\title{
Multi-Variable Self-Tuning Feedback Linearization Controller for Power Oscillation Damping
}

\author{
Jawad Arif, Member, IEEE, Swakshar Ray, Member, IEEE, and Balarko Chaudhuri, Senior Member, IEEE
}

\begin{abstract}
The objective of this paper is to design a measurement based self-tuning controller which does not rely on accurate models and deals with nonlinearities in system response. A special form of neural network (NN) model called as feedback linearizable neural network (FLNN) compatible with feedback linearization technique is proposed for representation of nonlinear power systems behaviour. Levenberg-Marquardt (LM) is applied in batch mode to improve the model estimation. A time varying feedback linearization controller (FBLC) is employed in conjunction with the FLNN-LM estimator to generate the control signal. Validation of the performance of proposed algorithm is done through the modeling and simulating both normal and heavy loading of transmission lines, when the nonlinearities are pronounced. Case studies on a large scale 16-machine, 5-area power system are reported for different power flow scenarios, to prove the superiority of proposed scheme against a conventional model based controller. A coefficient vector $\Lambda$ for FBLC is derived and utilized online at each time instant, to enhance the damping performance of controller.
\end{abstract}

Index Terms-Self-tuning Control, Power Systems, Feedback Linearizable Neural Networks, Feedback Linearization Controller, Online Estimation.

\section{INTRODUCTION}

$\mathbf{E}$ LECTRIC power supply systems are large, complex and highly interconnected. In many parts of the world, deregulation, restructuring and continued uncertainties of what is yet to come has led utilities to make different investment choices. The process of gaining permission to construct new lines has become extremely difficult, expensive, and timeconsuming [1]. Also, with the increase of power flow through the tie-lines, the damping of inter-area modes degrade, exciting low frequency oscillations [2]; range: $0.1-1.0 \mathrm{~Hz}$. A number of incidents have been reported in the past due to such oscillatory instabilities [3]. Traditionally these oscillations are damped with the power system stabilizer (PSS), which provide a supplementary control action through the excitation control of the generator [4]. In recent years flexible ac transmission systems (FACTSs) devices [5] have proven effective in the damping of these oscillations where the supplementary control over voltage and power flow is exerted [6]. This ensures the better utilization of transmission system [7].

Power systems behaviour is highly nonlinear in nature. Under stressed operating conditions the nonlinear effects are

J. Arif is former PhD Student at Imperial College London, SW7 2AZ, UK e-mail: jawad.arif@imperial.ac.uk.

S. Ray is with GE Global Research, New York, USA e-mail: rays@ge.com.

B. Chaudhuri is Senior Lecturer in the Department of Electrical and Electronic Engineering, Imperial College London, SW7 2AZ, UK e-mail: b.chaudhuri@imperial.ac.uk. more prominent. Thus the key challenge is to design the nonlinear damping controller for the FACTS devices which takes account of nonlinearities and changes in operating conditions. Although, linear controllers are commonly designed to provide satisfactory performance around a single operating condition, their performance can be enhanced using robust control techniques [8]-[10]. However, following severe contingencies, the post-contingency system can be different from its nominal operating states and even beyond the performance radius of designed robust controllers. Thus, a self-tuning controller which takes account of the nonlinearities in system and adapts to the changes in operating conditions could potentially yield better results [11]. This controller relies solely on measured signals and has been proposed for PSSs [12] and FACTS devices [13], working in linear domain. A practical problem for any adaptive/self-tuning controller is that it does not have a nominal representation and hence is difficult to include in the nominal model for system studies. However, because of their self-tuning nature, they are expected to adapt/self-tune to the situation even though they were not considered in a study. For example, a conventional PSS designed without the knowledge of another adaptive/self-tuning PSS would work together in unison as the latter would have the information about updated (due to inclusion of the conventional PSS) dynamics at each sampling instant.

Nonlinear model and the correct estimation of the model parameters is vital for the representation of power system dynamics, especially under stressed operating conditions. Mostly, various neural networks models are used by researchers [14][19] for the accurate representation of the nonlinear systems which has the advantage of its generalization and learning ability [18]. In recent years, use of multi-layer perceptron (MLP), radial basis function (RBF), recurrent and simultaneous recurrent neural network (RNN and SRN) has been reported for online estimation of input-output mapping of nonlinear systems [18], [20]-[22]. These methods typically use back-propagation (BP) or back-propagation through time (BPTT) to update online the neural network parameters. However, the learning process of back propagation algorithm is slow [23], and they have limitations in terms of convergence time and accuracy [18], [20], [21]. In this work, an online Levenberg-Marquardt (LM) [24]-[27] algorithm is adopted to be used with nonlinear neural networks ensuring better accuracy and convergence. In our investigation of online estimation, the classical LM is adapted to work in sliding window batch mode.

Neural network based control in linear/nonlinear form have been proposed in power systems [11], [16], [18], however, 
without any analytical proof of closed loop stability. In linear control, the pole shifting controller is employed [28] to emphasize on the stability of the closed loop system, which is based on the pole characteristics of polynomial. However, it is not easy to choose suitable closed loop pole locations, especially if the system operates over a wide ranging conditions [29]. Hence a nonlinear controller, Feedback linearizable controller $(F B L C)$ [30]-[34] is essential to overcome this problem. In this work, a special form of nonlinear neural network called as feedback linearizable neural network (FLNN), compatible with the FBLC, is used to represent the nonlinear low frequency dynamics of the system.

In this paper a modified neural network (FLNN) is used with an online LM [27] algorithm in conjunction with the feedback linearization controller [31]-[34] to damp oscillations. This work explores the self-tuning controller for TCSC applications in multi-variable frame-work i.e., multi-input, single-output (MISO). In [28], the FBLC has been used to damp single modal oscillation using a single-input, single-output (SISO) controller. It is observed that multiple modes are difficult to handle with SISO in large power systems as the observability of all the dominant modes are not necessarily adequate at a single location.

The multi-input, multi-output (MIMO) system has advantages for the choice of controller design, and is effective once multiple actuators (TCSCs) are installed in large scale power systems rather than uncoordinated design of individual SISO controller for each actuator. Here, the measured signals from the different geographical locations are used in estimator and controller to generate the control signals for the TCSCs. However, this situation leads to the long distance transmission of the control signals to remote geographical locations, which is not practical in the power systems. A further step to reduce the susceptibility of multiple control signals by replacing with only one controller output i.e., MISO. The MISO scheme is viable if there is a single actuator installed in the power system.

The overall research intent is to design a generic nonlinear MISO self-tuning controller which damp the oscillations in the power systems for various post-disturbance system operating conditions without manual adjustment or re-tuning of controller parameters. Moreover, it should require minimal a priori knowledge about the system and post-disturbance system operating conditions. The major contributions of this work can be summarized as follows:

- Extend the classical LM algorithm to sliding window batch mode for online estimation of power system dynamic behaviour.

- Construct an appropriate MISO FLNN structure compatible with the FBLC framework.

- Ascertain an adaptive coefficient vector $\Lambda$ suited for FBLC in changing operating conditions (post disturbance).

- Demonstrate and compare MISO FBLC for power systems application under normal and high power flow scenarios when nonlinearities are pronounced.

The performance of proposed FLNN with LM along with the FBLC is tested on a 16-machine 5-area test system [35] under normal and heavy power flow transfer over the tie-lines.

\section{STUdy System}

The study system considered for the case study has 16 machines and 68 buses distributed over 5 different geographical areas as shown in Fig. 1. This is a reduced equivalent of the New England (NETS) - New York (NYPS) interconnected system from the seventies with three neighbouring areas (Areas 3,4 and 5) represented by their dynamic equivalents. All the generators are represented by sub-transient models. Generators G1-G8 have DC1A exciters, G9 has a static exciter with a power system stabilizer (PSS) while the rest, G10-G16, are under manual excitation control [4]. A detailed description of the study system including the generator, excitation system, load and network parameters can be found in [35].

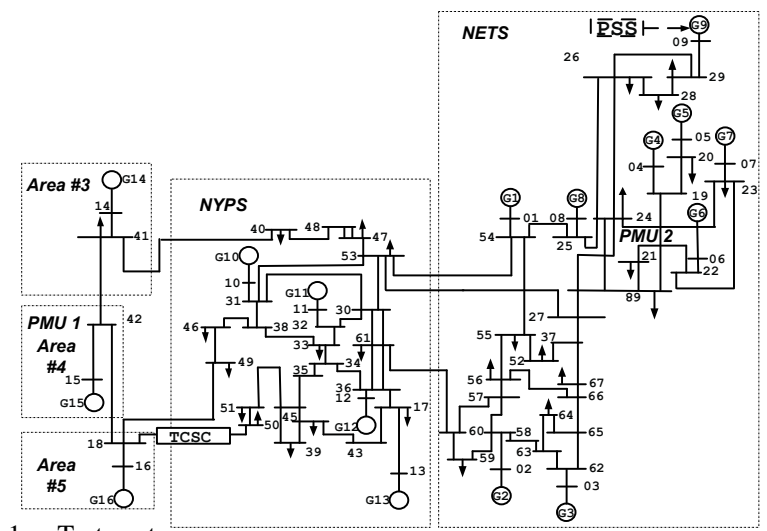

Fig. 1. Test system.

Under nominal condition, about $1500 \mathrm{MW}$ and $600 \mathrm{MW}$ are exported to the NYPS from areas 3 and 5 , respectively. To support these large power transfers, a thyristor controller series capacitors (TCSC) is installed in the transmission lines connecting buses $18-50$. In steady-state, this TCSC provides $50 \%$ (of line reactance) capacitive compensation. Modal analysis reveals the presence on three critical low frequency oscillatory modes (approximately $0.35,0.50$ and $0.60 \mathrm{~Hz}$ ) with poor (less than $4 \%$ ) damping.

The reasonably stress operating conditions were considered in this paper (large power flow between areas) where the nonlinearities were more pronounced. Active power flow through the lines connecting buses $45-35$ and $16-18$ were found to be the most appropriate feedback signals for reasons mentioned in [35], [36]. This results in a 2-input and a single output control structure.

The aim of this paper is to demonstrate potential application of multi-variable self-tuning feedback linearization controller for damping inter-area oscillations in power systems. The proposed control algorithm is general and applicable to any power systems actuator including series or shunt FACTS devices, high-voltage, direct current (HVDC) links etc. Of course the controllability of the inter-area modes will depend on the type and the location of the actuators. Here, the choice of TCSC was to control the large power transfer between Area 5 and NYPS.

\section{Conventional Control (CCL) Design}

The design methodology for a low order $n_{c c l}$-input, single output MISO controller is described in a general form in 
this section. It is assumed that there are ' $n_{c c l}$ ' number of critical inter-area modes which are highly observable from ' $n_{c c l}$ ' different locations. The controller is made up of only gain $\left(K_{1}, K_{2}, \ldots K_{n_{c c l}}\right)$ and a maximum of three first order lead-lag blocks in each channel apart from the standard low pass noise filters $\left(T_{m}=0.01 \mathrm{~s}\right)$ and the high-pass washout blocks $\left(T_{w}=10.0 \mathrm{~s}\right)$. Measured signals are denoted as $P_{1}$, $P_{2}, \ldots P_{n_{c c l}}$ and the control signal to the actuating TCSCs as $u$ 's in [37].

The controller is required to ensure that oscillations settle within specific time for all the operating conditions. The design specifications of such a controller are as follows:

1) Modal oscillation due to all the poorly damped interarea modes should settle within $10 \mathrm{~s}$ for all the operating conditions considered during design.

2) Frequency of oscillation of the inter-area modes should not change appreciably from their open-loop values.

3) Controller effort should be optimum.

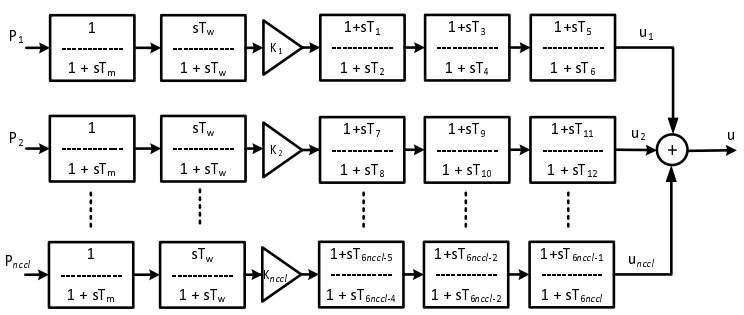

Fig. 2. General block diagram of $n_{c c l}$-input, 1 -output controller.

An objective function with constraints for these three design specifications is formulated in [37]. The multi-objective constrained optimisation problem can be solved using any standard optimisation technique. However, it is not straight forward to come up with a reasonable initial guess for the parameter set. Hence, evolutionary optimisation techniques (e.g. genetic algorithm, particle swarm optimisation etc.) have been found to be more effective in terms of convergence. The results presented is this paper were obtained using a particle swarm optimisation (PSO) technique [38].

A comparison between CCL and proposed controller has been reported in Section VII. The number of inputs $n_{c c l}$ is 2 in this conventional control design.

\section{ESTIMATION OF MISO FEEDBACK LINEARIZABLE NEURAL NETWORK (FLNN) USING LM ALGORITHM}

Feedback linearizable neural networks have a built-in capability to adapt their synaptic weights according to the change in the system response. In particular, a FLNN trained to operate in a specific environment can be easily retrained to deal with minor changes in the operating conditions. The FLNN-based identification technique aims to develop neural network models for a power systems, operating under different conditions using input - output data. The main advantage of the $\mathrm{NN}$-based technique comes from the efficient implementation of this model. The FLNN model parameters, synaptic weights, can be computed iteratively so as to attain a desired design objective. The updates of synaptic weights are done through the LM algorithm which is adopted in online batch mode.

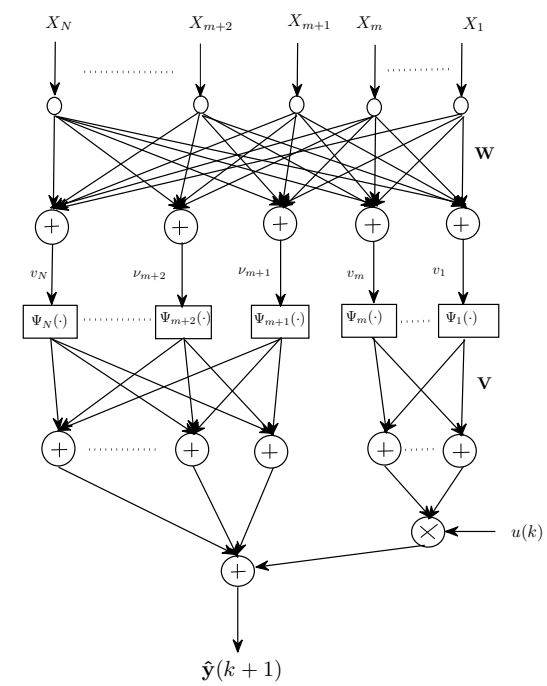

Fig. 3. Modified structure of conventional neural network: Feedback Linearizable Neural Network (FLNN).

\section{A. MISO Structure of Feedback Linearizable Neural Network $(F L N N)$}

The recurrent neural network based identification methodology provides an efficient and cost effective alternative to the existing modeling techniques [39]. The future dynamic behaviour can be estimated by using the current and past information of input-output. But the non-compatibility of a recurrent network with classical nonlinear control framework is a problem. To overcome this, a nonlinear feedback linearizable neural network (FLNN) for MISO framework is adopted in this paper, as shown in Fig. 3.

The estimated output of the FLNN is meant to track the following nonlinear model (1) and the estimated $\hat{\mathbf{f}}(X)$ and $\hat{\mathrm{g}}(X)$ are used to generate the control signals from FBLC. The proposed form of FLNN is:

$$
\hat{\mathbf{y}}(k+1)=\hat{\mathbf{f}}(X)+\hat{\mathbf{g}}(X) \times u(k)
$$

where

$\hat{\mathbf{f}}(X)=\left[\begin{array}{c}\sum_{i=m+1}^{N}\left[V_{1 i} \times \Psi_{i}\right] \\ \sum_{i=m+1}^{N}\left[V_{2 i} \times \Psi_{i}\right] \\ \vdots \\ \sum_{i=m+1}^{N}\left[V_{n_{p}} \times \Psi_{i}\right]\end{array}\right], \hat{\mathbf{g}}(X)=\left[\begin{array}{c}\sum_{i=1}^{m}\left[V_{1 i} \times \Psi_{i}\right] \\ \sum_{i=1}^{m}\left[V_{2 i} \times \Psi_{i}\right] \\ \vdots \\ \sum_{i=1}^{m}\left[V_{n_{p} i} \times \Psi_{i}\right]\end{array}\right]$

where $N \triangleq m+n_{p} n . \hat{\mathbf{g}}(X) \in \mathbb{R}^{n_{p}}$ is a column vector.

$m$ : no of previous control inputs, $n$ : no of previous measurements, $n_{p}$ : no. of measurement signals and $N$ : no of neurons. Also, $X=[\bar{u}, \bar{x}]^{\mathrm{T}}$,

$$
\begin{aligned}
& \bar{x}=\left[y_{1}(k-n+1), y_{1}(k-n+2), \cdots, y_{1}(k), \cdots\right. \\
& \left.y_{n_{p}}(k-n+1), y_{n_{p}}(k-n+2), \cdots, y_{n_{p}}(k)\right] \in \mathbb{R}^{n_{p} n}
\end{aligned}
$$

is the matrix of past $n-1$ and the current measurement of each $y$. Also,

$$
\bar{u}=[u(k-m), u(k-m+1), \cdots, u(k-1)] \in \mathbb{R}^{m}
$$

is the matrix of $m$ previous control inputs of each $u$. $\Psi($.$) can$ be chosen based on the Kolmogorov's theorem for universal 
approximation [40]. Here, $\Psi_{i}\left(v_{i}\right)=\frac{1}{1+e^{-\left(v_{i}\right)}}$ and

$$
v_{i}=\sum_{j=1}^{N}\left(W_{i j} \times X_{j}\right), \quad i=1,2, \cdots, N .
$$

The estimated output of the neural network with a single hidden layer can be written as:

$$
\hat{\mathbf{y}}(k+1)=\mathbf{V} \times[\mathbf{u} \times \Psi(\mathbf{W} \times X)] \in \mathbb{R}^{n_{p}}
$$

where, $\mathbf{u}=\operatorname{diag}[u(k), u(k), 1 \cdots 1]$ is a diagonal matrix of size $\mathbb{R}^{N \times N}$.

The adjustment of FLNN parameters, $\mathbf{W} \in \mathbb{R}^{N \times N}$ and $\mathbf{V} \in \mathbb{R}^{n_{p} \times N}$, are done iteratively with online LM algorithm which provide faster detection of oscillatory behaviour of a system after disturbances under varying operating conditions. The training of the FLNN is discussed in the next subsection.

\section{B. Estimation of FLNN Parameters using LM Algorithm}

A modified Levenberg-Marquardt(LM) algorithm is presented here to train the FLNN. Like the Gauss-Newton methods, the LM algorithm was designed to approach secondorder training speed without having to compute the Hessian matrix [26]. Mostly, it is used for off-line batch training of different neural networks. Use of LM algorithm for estimating the parameters of the neural network leads to faster convergence [27], [24]. This algorithm was used for estimation of 4-machines, 2-area power system [28] and 16-machines, 5 -area power system with MIMO configurations. In this paper, this algorithm successfully estimates the power system behaviour with normal and high power flow where nonlinearities are more pronounced.

As in [28], the conventional LM algorithm is adapted here for FLNN to work in sliding window batch mode. A suitable window size is first selected such that it covers half to one cycle of the lowest frequency $(0.35 \mathrm{~Hz})$ oscillatory mode.

The error vector $\bar{e}$ over a window containing $w_{s}$ samples is given by:

$$
\bar{e}_{i}=\left[\begin{array}{c}
y_{i}(k)-\hat{y}_{i}(k) \\
\vdots \\
y_{i}\left(k-w_{s}+1\right)-\hat{y}_{i}\left(k-w_{s}+1\right)
\end{array}\right] \in \mathbb{R}^{w_{s}},
$$

where $i=1,2, \cdots, n_{p}$. Also, $y(\cdot)$ is the actual output, $\hat{y}(\cdot)$ is the estimated output and $w_{s}$ is the number of samples in a window. The overall error vector is

$$
\mathbf{e}=\left[\begin{array}{cccc}
\bar{e}_{1}^{\mathrm{T}} & \bar{e}_{2}^{\mathrm{T}} & \cdots & \bar{e}_{n_{p}}^{\mathrm{T}}
\end{array}\right]^{\mathrm{T}} \in \mathbb{R}^{n_{p} w_{s}}
$$

To calculate the error derivatives over an entire window the weights to be updated (unknown parameters) are arranged in a form of a vector $\bar{p}$ as follows:

$$
\bar{p}=\left[\begin{array}{llllllll}
W_{i 1}^{\mathrm{T}} & W_{i 2}^{\mathrm{T}} & . \cdot & W_{i N}^{\mathrm{T}} & V_{i 1}^{\mathrm{T}} & V_{i 2}^{\mathrm{T}} & . . & V_{i n_{p}}^{\mathrm{T}}
\end{array}\right]^{\mathrm{T}},
$$

where $i=1,2, \cdots, N$.

The total number of unknown parameters is $N_{p}=N(N+$ $n_{p}$ ) which is the size of the parameter vector $\bar{p}$. A good strategy to select learning rate $\chi_{k}$ is that, initially, a small value is chosen and if this step does not yield the smaller value in $\mathbf{e}^{\mathrm{T}} \mathbf{e}$, then the step size is further increased to a factor $\rho>1$. This leads to decrease in $\mathbf{e}^{\mathrm{T}} \mathbf{e}$ due to a small step in descent direction. If a step produces minimum $\mathbf{e}^{\mathrm{T}} \mathbf{e}$, then $\chi_{k}$ is divided by $\rho$ in the subsequent step [28]. This translates the LM algorithm into a Gauss-Newton method. Thus the LM algorithm results into a good compromise between the speed gained through the Newton method and guaranteed convergence of steepest descent method [27]. The weight parameters are then updated according to (9) [27].

$$
\bar{p}_{\text {new }}=\bar{p}_{\text {old }}+\left[\mathbf{J}^{\mathrm{T}} \mathbf{J}+\chi_{k} I\right]^{-1} \mathbf{J}^{\mathrm{T}} \mathbf{e} \in \mathbb{R}^{N_{p}}
$$

The parameter update is done online for each moving window of size 35 . A new sample is added into the window either the product $\mathbf{J}^{\mathrm{T}} \mathbf{e} \geq 10^{-9}$ or the numbers of iterations for a window reach to 30 . The parameter vector $\bar{p}_{\text {new }}$ is updated after the inclusion of new sample into the window. This scheme helps to avoid infinite loop in the batch processing of LM algorithm.

\section{MISO FEEDBACK LINEARIZATION CONTROL (FBLC)}

In the feedback linearization approach, the objective is to linearize the map between the transformed inputs $v$ and the actual outputs $\mathbf{y}$. This design is mostly used in the tracking problem. But in this work, feedback linearization controller is designed to regulate the output signal $\mathbf{y}$ to track $\mathbf{y}_{\mathbf{d}}=$ $\left[y_{d_{1}}(k) \quad y_{d_{2}}(k) \cdot \cdot y_{d_{n_{p}}}(k)\right]$ through the bounded control action $u$. The feedback linearizable controller is of the order: $\mathbf{y} \in \mathbb{R}^{n_{p}}$ and $u \in \mathbb{R}$. The envelop of the desired trajectories $\mathbf{x}_{d}$ and system output $\mathbf{y}_{s}$ for a window is defined as:

$$
\begin{array}{r}
\mathbf{x}_{d}(k) \triangleq\left[\begin{array}{cccc}
\mathbf{y}_{\mathbf{d}}^{\mathrm{T}}(k-n+1) & \cdots & \mathbf{y}_{\mathbf{d}}^{\mathrm{T}}(k-1) & \mathbf{y}_{\mathbf{d}}^{\mathrm{T}}(k)
\end{array}\right]^{\mathrm{T}} \\
\mathbf{y}_{s}(k) \triangleq\left[\begin{array}{cccc}
y_{1}(k-n+1) & \cdots & y_{1}(k-1) & y_{1}(k) \\
\vdots & \cdots & \vdots & \vdots \\
y_{n_{p}}(k-n+1) & \cdots & y_{n_{p}}(k-1) & y_{n_{p}}(k)
\end{array}\right]^{\mathrm{T}}
\end{array}
$$

The assumption is that the $\mathbf{g}(X) \neq 0$. This is a necessary condition for the existence of solutions for (2). The error vector is defined as

$$
\mathbf{e}(k) \triangleq \mathbf{y}_{s}(k)-\mathbf{x}_{d}(k) \quad \in \mathbb{R}^{n \times n_{p}}
$$

and a filter error $\mathbf{r} \in \mathbb{R}^{n_{p}}$ as:

$$
\begin{aligned}
& \mathbf{r}^{\mathrm{T}}(k) \triangleq\left[\Lambda^{\mathrm{T}}(k) 1\right] \mathbf{e}(k), \\
& \text { where, } \quad \Lambda(k)=\left[\begin{array}{llll}
\lambda_{1} & \lambda_{2} & \ldots & \lambda_{n-1}
\end{array}\right]^{\mathrm{T}}
\end{aligned}
$$

is appropriately chosen coefficient vector such that $\mathbf{e}(k) \rightarrow 0$ as $\mathbf{r}(k) \rightarrow 0$ (i.e. $z^{n-1}+\lambda_{n-1} z^{n-2}+\cdots+\lambda_{1}$ is stable).

It is noted that the choice of $\Lambda$ dictates the performance of FBLC. The usual way is to keep the $\Lambda$ constant, resulting $\mathbf{e}(k) \rightarrow 0$ as $\mathbf{r}(k) \rightarrow 0$. But due to change of operating conditions, a fixed value for $\Lambda$ does not provide acceptable performance for the current application. The alternative way to determine the $\Lambda$ is through the self-tuning scheme, which updates the $\Lambda$, at each time instant $k$ (refer Fig. 4). This proposed scheme makes the FBLC as a time varying controller. The formulation of self-tuned $\Lambda$ is described next in section $\mathrm{V}-\mathrm{A}$.

Equation (1) can be written in term of filtered error as:

$$
\mathbf{r}(k+1)=\mathbf{f}(X)+\mathbf{g}(X) u+\mathbf{Y}_{d} \in \mathbb{R}^{n_{p}}
$$

where $\mathbf{f} \in \mathbb{R}^{n_{p}}, \mathbf{g} \in \mathbb{R}^{n_{p}}$ and

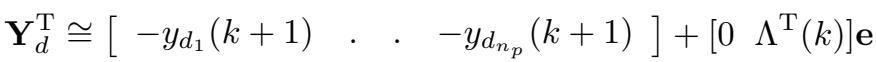




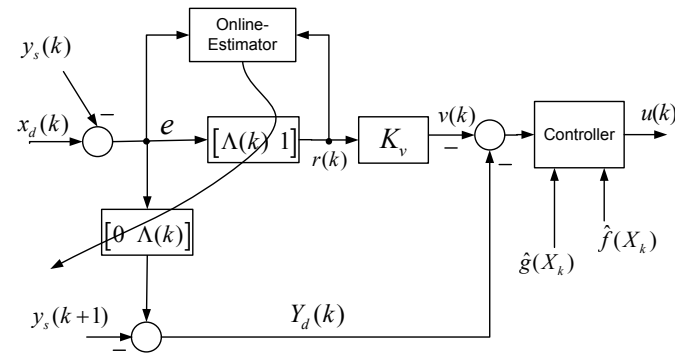

Fig. 4. Structure of the discrete time feedback linearization time-varying controller.

In this paper, we assumed that $\mathbf{g}(X)$ is a non-zero column vector. Thus, the inverse of $\mathrm{g}(X)$ does not exist and it does not guarantee the exact solution of (1). An pseudo-inverse approach [41] is used which provides a least squares solution demonstrated in (14):

$$
u=\left[\mathbf{g}^{\mathrm{T}}(X) \mathbf{g}(X)\right]^{-1} \mathbf{g}^{\mathrm{T}}(X)\left[-\mathbf{f}(X)-K_{v} \mathbf{r}-\mathbf{Y}_{d}\right]
$$

would bring $\mathbf{r}(k)$ to zero for $0 \leq K_{v}<1$ [42]. Since it is assumed that these functions are estimated by the LM algorithm, the choice of control law can be given as:

$$
u=\left[\hat{\mathbf{g}}^{\mathrm{T}}(X) \hat{\mathbf{g}}(X)\right]^{-1} \hat{\mathbf{g}}^{\mathrm{T}}(X)[-\hat{\mathbf{f}}(X)+\nu]
$$

where the estimates $\hat{\mathbf{f}}(X)$ and $\hat{\mathbf{g}}(X)$ will be constructed by neural network, and the auxiliary term is $\nu=-K_{v} \mathbf{r}-\mathbf{Y}_{d}$. It can be noted that the control law (15) is not well defined when the $\hat{\mathbf{g}}^{\mathrm{T}}(X) \hat{\mathbf{g}}(X)$ is singular. Simple approaches to solve this problem are: keep the estimate $\hat{\mathrm{g}}$ constant. This limits the class of systems and gets affected depending on its bound. Or reconstruct $\hat{\mathrm{g}}$ by an adaptive scheme. A local solution can be given, but it is not easy to select initial weights, so that the output of $\mathrm{NN}$ is satisfactorily approximated.

Another way to keep $\hat{\mathrm{g}}^{\mathrm{T}}(X) \hat{\mathrm{g}}(X)$ non-singular is through the weight adaptation law as proposed and applied in this paper. The structure of $\mathrm{NN}$ and adaptive law to update the weights of $\mathrm{NN}$ has been modified to overcome the problem described in [14], [28].

The initial weights in Fig. 3 are chosen as a small positive numbers. The input weights $\mathbf{W}$ are updated at each time step through the LM algorithm. The output weights related to $\mathbf{f}(X)$ in (5) are updated at each time instant while the weights related to $\mathrm{g}(X)$ are fixed from start of the estimation. This ensures that the $\mathbf{g}(X)$ remains $>0$ throughout the estimation process.

\section{A. Self-tuning Technique for $\Lambda(k)$}

The operating conditions are changed frequently in power systems due to increase in loads or line contingency. Therefore, the time-invariant controller does not perform well. Here in this work, a modification has been made in FBLC which lead it to a time-varying controller. The self-tuning approach is used to update the $\Lambda$ at each time step by using the projection algorithm. The coefficient vector is adjusted online according to the change in operating condition/load. Let

$$
\begin{aligned}
& \mathcal{E} \triangleq\left[\begin{array}{llll}
e_{1}(k) & e_{2}(k) & \cdots & e_{n_{p}}(k)
\end{array}\right]^{\mathrm{T}} \quad \text { and } \\
& \varphi^{\mathrm{T}} \triangleq\left[\begin{array}{llll}
\mathcal{E}(k-n+1) & \mathcal{E}(k-n+2) & \cdots & \mathcal{E}(k-1)
\end{array}\right]
\end{aligned}
$$

Then (11) can be re-written as:

$$
\mathbf{r}(k)=\varphi^{\mathrm{T}} \Lambda^{*}+\mathcal{E}(k)
$$

If the true parameter vector $\Lambda^{*}$ is not known then an estimate of the $k^{t h}$ observation is $\hat{\mathbf{r}}(k)=\varphi^{\mathrm{T}}(k-1) \Lambda(k)+\mathcal{E}(k)$. Where $\Lambda(k)$ is an estimate of $\Lambda^{*}$. Now the cost function can be written as:

$$
\begin{array}{r}
J_{\kappa}(\Lambda(k), \Lambda(k-1), k)=\frac{1}{2}\|\Lambda(k)-\Lambda(k-1)\|_{2}^{2} \\
+\kappa^{\mathrm{T}}\left[\mathbf{r}(k)-\varphi^{\mathrm{T}}(k-1) \Lambda(k)-\mathcal{E}(k)\right] \in \mathbb{R}
\end{array}
$$

which is quadratic based on the a-priori performance of $\varphi^{\mathrm{T}}(k) \Lambda(k) . \kappa \in \mathbb{R}^{n_{p}}$ is the Lagrange multiplier. To find the minimum of the cost function, the gradient of (17) with respect to $\kappa$ and $\Lambda$ is equated to zero [43] and [44, p. 52]. The projection estimate of $\Lambda(k)$ is given by:

$$
\begin{aligned}
& \Lambda(k)=\Lambda(k-1) \\
& \quad \gamma \mathcal{G} \varphi(k-1)\left[\mathbf{r}(k)-\varphi^{\mathrm{T}}(k-1) \Lambda(k-1)-\mathcal{E}(k)\right]
\end{aligned}
$$

where $\mathcal{G}=\left[\alpha I+\varphi(k-1) \varphi^{\mathrm{T}}(k-1)\right]^{-1}, \alpha \geq 0$ and $0<\gamma<2$. At each iteration $\Lambda(k)$ is updated before generating the control signals.

\section{Closed-Loop Stability}

With indirect adaptive control, the overall closed-loop stability is always an issue and analytical proof of stability is likely to be a significant contribution in the control community. Such an analytical proof of stability is beyond the scope of this paper. Nonetheless, three major steps were taken in this work to ensure the stability of overall system:

1. An adaptive technique is proposed in Section V-A to ensure the stability of FBLC controller. The error $\mathbf{e}(k)$ in (10) approaches zero as $\mathbf{r}(k)$ approaches zero. Moreover, the cost function in (17) aims to find the true parameters of coefficient vector of FBLC while taking the error (10) into consideration.

2. A new approach is introduced in FBLC to overcome the problem of singularity which is explained in the paragraphs following (15). The idea is to ensure that inverse of $\mathbf{g}^{\mathrm{T}}(X) \mathbf{g}(X)$ remain nonsingular through the weight adaption law. This show the flexibility and adaptability of proposed FLNN.

3. An effort has been done with the offline LM algorithm to work in online batch mode while assuring the convergence of weights parameters of FLNN model. A loop is iterated till the error between actual and estimated output is less than some defined $\varepsilon_{1}-$ refer LM algorithm in [28]. This is third step to make sure the stability of the proposed control scheme.

\section{CASE STUdy}

A case study involving a typical power system problem is presented in this section to highlight the effectiveness of the proposed FBLC approach.

The proposed approach is not model based in the sense that the controller design does not rely on accurate and updated model which requires information about all the power system components and operating conditions. With minimal apriori knowledge (e.g. number of dominant modes etc) the 
proposed controller can produce the desired damping and adapt according to change in operating conditions. The FLNN structure allows proper handling of nonlinearities in system dynamic response which could be prominent under stressed operating conditions. However, the proposed control algorithm is computationally more intensive than a classical controller. With fast computers available these days, this is not necessarily a limitation.

\section{A. Design Parameters Used in Simulation Study}

For the current application, there are three dominant interarea modes in the system, therefore, the number of previous values of the measurement signals (i.e. the system order) was chosen as $n=7$. Normally, a second order system is good enough to identify the single mode in a system. Based on this experience, the seventh order model is chosen to identify the three critical inter-area modes, present in this particular test system. An order of 7 ensured that the lowest frequency modes were only identified by the neural network. Due to the $7^{\text {th }}$ order of FLNN, the number of hidden layer neurons to be $N=21$. Other parameters used for the simulation are: $n_{p}=$ 2 measurement signals, a control input and their past values $m=7$ with $w_{s}=35$. The FLNN structure used logsigmoid function in the hidden layer, therefore, it was necessary to scale the inputs of the neural network between +1 and -1 . This was to ensure that the inputs to the logsigmoid function do not fall in the saturation region. For the need of faster convergence, the learning rate was selected as $\chi=0.1$ which was found suitable for training the neural network with minimum undershoot and overshoots. The initial coefficient $\Lambda$ vector $(0.35 \pm 0.045 i$, $0.22 \pm 0.041 i, 0.14 \pm 0.034 i$ ) in FBLC have been tested for multiple conditions and multiple disturbances and have shown satisfactory performances for all operating scenarios.

Simulations were carried out in Matlab/SIMULINK to demonstrate the performance of proposed control structure for different contingencies. A fixed step size of $1.0 \mathrm{~ms}$ was used while the feedback signals were sampled at $50 \mathrm{~Hz}$ according to the standard sampling rate of practical phasor measurement units (PMUs). Several scenarios were considered under different loading conditions to validate the performance and robustness of the proposed control algorithm. These scenarios are shown in Figs. 5 - 7 including the location of the fault and line outage. Amongst these test scenarios, first case relates to the rated power flow in the transmission line, where 3 -phase faults for $80 \mathrm{~ms}$ persists, described next in subsection Test Case VII.1. Further two cases are tested with $10 \%$ increased power flow in the transmission to demonstrate the capability of the proposed controller, explained in subsection Test Case VII.2. The system response is shown in terms of the measured active power flow in buses $16-18$ and 45-35 ( $P_{\text {line }_{16-18}}$ and $P_{\text {line }_{45-35}}$ ), and the difference between the rotor angle of generators in different geographical areas. Low frequency oscillatory modes involving generators and loads across diverse geographical areas are expected to be predominantly visible in these signals. Also the dynamic variation of the compensation, $K_{c}$ by the TCSC is shown for each scenario.
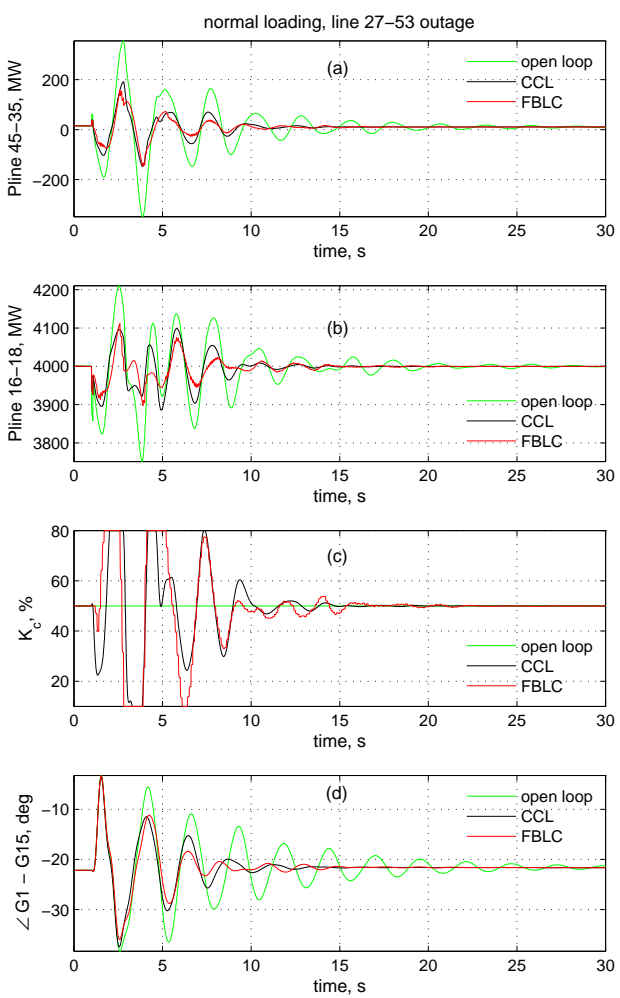

Fig. 5. Response of the TCSC control signals due to a three phase fault at bus 27 followed by line $27-53$ outage.

\section{B. Normal Power flow Conditions}

Test Case VII.1. The performance of a conventional controller is bench-marked against the proposed FBLC in the backdrop of the open-loop (no supplementary control of the TCSC) response of the system. In each Figure (5-7), the first two subplots (' $a$ ' and ' $b$ ') depicts the measured power flow in the line $45-35$ and $16-18$. The third subplot ' $c$ ' shows the variation in the percentage compensation of the TCSC and the final subplot ' $d$ ' exposes the angular separation between the generator angle G1-G15 or G16-G13.

It is clear from Fig. 5 that FBLC yields equivalent performance of a CCL and both of these controllers are better than the open-loop system, as expected. The oscillations settle down within $8-10$ s for both the FBLC and CCL unlike the open-loop extended response time of beyond 15s. Here, FBLC works as good as the standard CCL for any scenario with very little knowledge of the accurate system model and related post-fault conditions. Whereas, in the designing of a CCL an accurate system model and tailored tuning is required for every new power flow condition.

Figure 5 illustrates the comparison of generator angles and the variation of percentage compensation of TCSC, $K_{c}$ between FBLC and CCL. Up to about $6-7 \mathrm{~s}$ the variation in $K_{c}$ is large due to the time taken by LM algorithm to estimate the accurate set of weight parameters of the FLNN.

So far we have seen the effectiveness of the proposed FBLC for a rated power flow scenario and proved its superiority to a conventional model based controller. This is due to its independence from the requirement of a system model and essential tuning of control parameters for specific scenarios. 

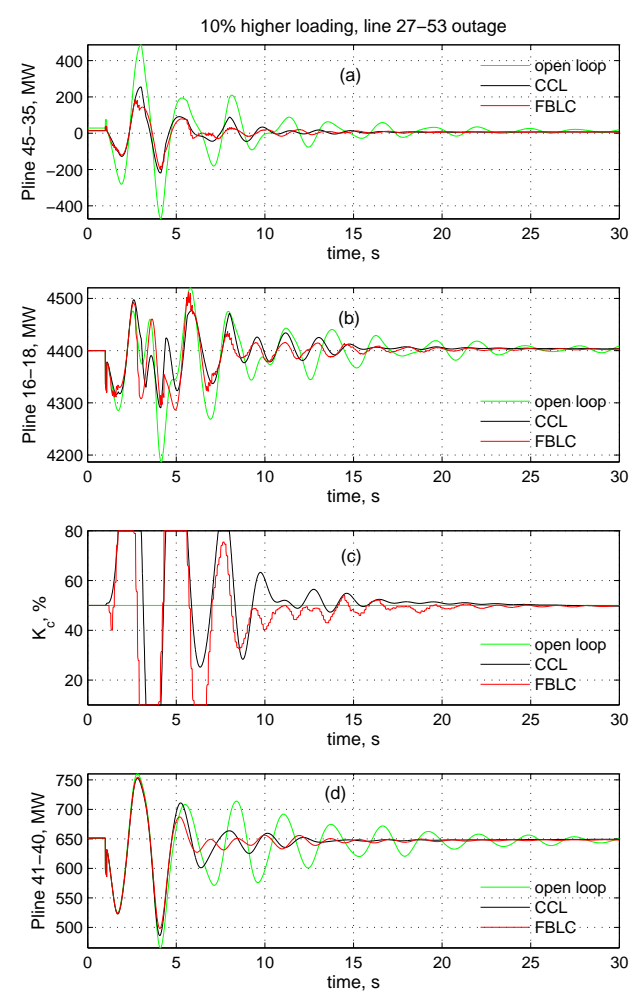

Fig. 6. Response of the TCSC control signals due to a three phase fault at bus 27 followed by line $27-53$ outage with $10 \%$ increase in load.

\section{Higher Power flow Conditions}

Test Case VII.2. In this test case, the higher nonlinearities in the system is introduced with the deliberate rise of $10 \%$ power flow in tie lines and performance of proposed FBLC structure is consequently observed. The design parameters for FBLC are described in VII-A, and the design of competitor conventional controller takes into account all outage scenarios. Please refer to Section III to find out the method of calculating the design parameters of conventional controller. The chosen simulation time is fixed to $30 \mathrm{~s}$. The results of various test scenarios is depicted in Figs. 6 and 7 where closed loop system responses (FBLC and CCL) under increased tie-line power flow can be seen against the open loop system.

In Fig. 7, $40-41$ line outage case, the conventional controller did not properly converge and yielded the unstable closed loop response. On the other hand the FBLC demonstrated superior performance over the CCL in both cases. The designed FBLC damps the oscillations within the minimum time of $10-12 \mathrm{~s}$, with the added advantage of ignoring the cumbersome step of manual redesign of model for each outage scenario - a step necessary for CCL. Here, LM algorithm estimates the parameters of FLNN at each time step and passes the estimated output, $\hat{\mathbf{f}}(X)$ and $\hat{\mathbf{g}}(X)$ to the controller. On the basis of these parameters the FBLC adjusts the coefficient vector at each time step such that the measured output successfully follows the desired output.

In Fig. 7(a) the active power through line $45-35$ located within the NYPS is shown while Fig. 7(d) shows the angular difference (i.e. power angle) between Area 5 (which includes G16) and NYPS (which includes the slack generator, G13).
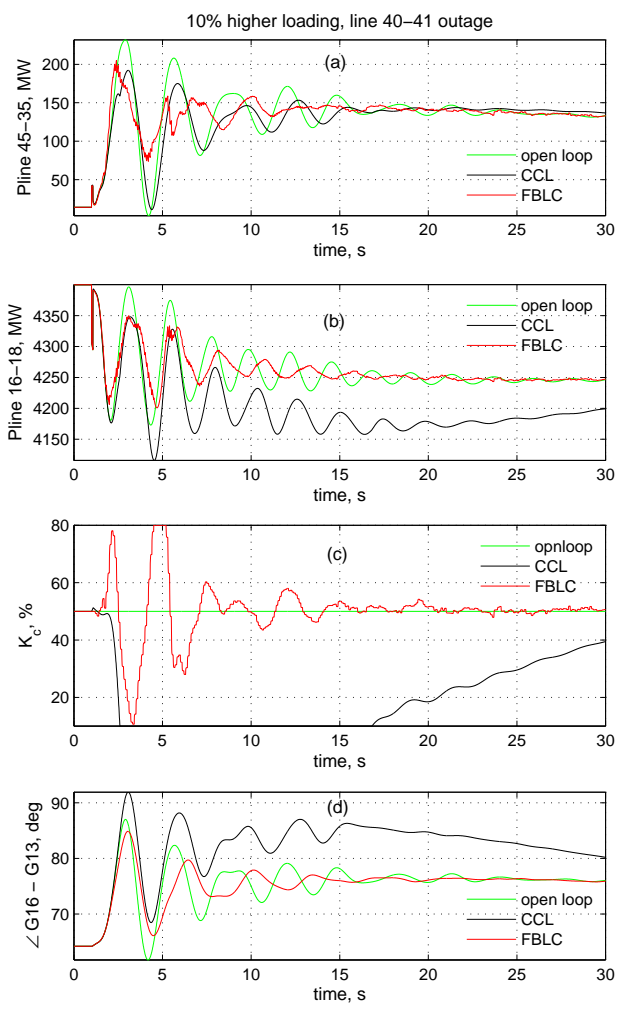

Fig. 7. Response of the TCSC control signals due to a three phase fault at bus 40 followed by line $40-41$ outage with $10 \%$ increase in load.

The modal contents are different for these two signals as the three critical inter-area modes are observable with varying degrees in these signals. A low frequency controller mode which takes long time to settle is seen in the angular difference between G16 and G13 is not observable in $P_{\text {line }_{45-35} \text {. }}$.

The angular separation between machine G16 and G13 located in different areas is shown in part ' $d$ ' of Fig. 7. Interarea oscillations involve a group of machines in one area swinging against a group of another area. These oscillations are mostly manifested in angular differences and therefore, chosen for the display. In each scenarios, the designed control scheme for TCSCs are capable to settle the oscillations within $10-15 \mathrm{~s}$ for a range of post-fault scenarios.

It is concluded in the case of higher non-linearities, the FBLC gives satisfactory performance for several operating conditions without the need of individual tuning of parameters. But it is necessary to modify the parameters in designing CCL whenever loading condition changes.

\section{CONCLUSIONS}

The paper presents four aspects of a measurement based control design in MISO framework for power oscillations damping control application.

- Nonlinear model estimation of the low frequency oscillatory dynamics using online LM algorithm with sliding window batch mode has been implemented. The adaptation of the modified LM algorithm for online application shows faster convergence and accurate estimation of the nonlinear dynamics. 
- Feedback linearization control is applied using the derived model. Due to the adaptation of the parameters, the system model gets updated in the post-disturbance operating conditions. Therefore, the adaptive FBLC controller shows satisfactory performance for various operating conditions without any need for manual adjustments.

- The major advantage of the proposed method is that it requires minimal a-priori knowledge about the system and post-disturbance system operating conditions.

- The FBLC is more effective in dealing with the stressed operating conditions e.g., high tie-line transfer, where the non-linearities are more pronounced.

The performance of FBLC is compared with the CCL under the normal and higher power flow conditions. The proposed FBLC gives sound performance than the conventional controller in all test cases. The results produced favour the real time implementation of FBLC in the industrial environment.

\section{REFERENCES}

[1] X. H. Chao, "System impact studies for dg projects under development in the us," IEEE Power Engineering Society Summer Meeting, vol. 1, no. 2, pp. 772-774, 2001.

[2] M. Klein, G. Rogers, and P. Kundur, "A fundamental study of inter-area oscillations in power systems," Transactions on Power Systems, vol. 6, no. 3, pp. 914-921, 1991.

[3] J. Paserba, "Analysis and control of power system oscillation," Ciger special publication 38.01.07, vol. Technical brouchure 111, 1996.

[4] P. Kundur, Power systems stability and control. New York: McGrawHill, 2005.

[5] N. G. Hingorani, "High power electronics and flexible AC transmission system," IEEE Power Engineering Review, vol. 8, no. 7, pp. 3-4, 1988.

[6] E. V. Larsen, J. J. Sanchez-Gasca, and J. H. Chow, "Concepts for design of facts controllers to damp power swings," IEEE Transactions on Power Systems, vol. 10, no. 2, pp. 948-956, 1995.

[7] A. Edris, "FACTS technology development: an update," IEEE, Power Engineering Review, vol. 20, no. 3, pp. 4-9, 2000.

[8] H.-B. Chen, J.-K. Shiau, and J. Chow, "Simultaneous h-infinity suboptimal control design," American Control Conference, vol. 3, 1995.

[9] N. Hemati, J. Thorp, and M. Leu, "Robust nonlinear control of brushless dc motors for direct-drive robotic applications," IEEE Transactions on Industrial Electronics, vol. 37, no. 6, pp. 460 - 468, 2002.

[10] L. Lei and W. Hong-Rui, "Robust tracking control of rigid robotic manipulators based on fuzzy neural network compensator," International Conference on Machine Learning and Cybernetics, Hong Kong, vol. 1, pp. $550-555,2007$.

[11] D. K. Chatuvedi and O. P. Malik, "Experimental studies of generalized neuron based adaptive power system stablilizer," Soft Computing - A Fusion of Foundations, Methodologies and Applications, vol. 11, no. 2, pp. 149-155, 2006.

[12] S.-j. Cheng, Y. Chow, O. Malik, and G. Hope, "An adaptive synchronous machines stablizer." IEEE Transaction on Power Systems, vol. 1, no. 3, pp. 101-107, 1986.

[13] R. Sadikovic, P. Korba, and G. Andersson, "Self-tuning controller for damping of power system oscillations with FACTS devices," IEEE Power Engineering Society General Meeting, Montreal, 2006.

[14] J. Arif, N. Ray Chaudhuri, S. Ray, and B. Chaudhuri, "Online Levenberg-Marquardt algorithm for neural network based estimation and control of power systems," International Joint Conference on Neural Networks, Atlanta, Georgia, USA, pp. 199-206, 2009.

[15] D. K. Chaturvedi and O. Malik, "Generalized neuron-based adaptive pss for multimachine environment," IEEE Transaction on Power Systems, vol. 20, no. 1, pp. 358-366, 2005.

[16] P. K. Dash, S. Mishra, and G. Panda, "Damping multimodal power system oscillation using a hybrid fuzzy controller for series connected facts devices," IEEE Transaction on Power Systems, vol. 15, no. 4, pp. $1360-1366,2000$.

[17] D. Chaturvedi and O. Malik, "Experimental studies of a generalized neuron based adaptive power system stabilizer," Soft Computing - A Fusion of Foundations, Methodologies and Applications, vol. Volume 11 , no. 2 , pp. $149-155,2007$
[18] W. Liu, G. Venayagamoorthy, and D. Wunsch, "Adaptive neural network based power system stabilizer design," Proceedings of the Intrl. Joint Conference on Neural Networks, vol. 4, no. 1, pp. 2970-2975, 2003.

[19] P. Shamsollahi and O.P.Malik, "An adaptive power system stabilizer using on-line trained neural networks," IEEE Transaction on Energy Conversion, vol. 12, no. 4, pp. 382-387, 1997.

[20] S. Ray and G. K. Venayagamoorthy, "Nonlinear modified pi control of multi-module gcscs in a large power system," Conference Record of the IEEE Industry Applications Society, vol. 3, pp. 1345-1351, 2006.

[21] — , "Real-time implementation of a measurement-based adaptive wide-area control system considering communication delays," IET Generation, Transmission \& Distribution, vol. 2, no. 1, pp. 62-70, 2008.

[22] J. C. Patra, R. N. Pal, B. N. Chatterji, and G. Panda, "Identification of nonlinear and dynamical systems using functional link artificial neural networks," IEEE transaction on Systems, Man and Cybernetic, vol. 29, no. 2, pp. 254-262, 1999 .

[23] A. Novokhodko and S. Valentine, "A parallel implementation of the batch backpropagation training of neural networks," International Joint Conference on Neural Networks, IJCNN, vol. 3, pp. 1783-1786, 2001.

[24] D. Marquardt, "An algorithm for least squares estimation on nonlinear parameters," SIAM J. APPL. MATH, vol. 11, pp. 431-441, 1963.

[25] F. Jinyan and P. Jianyu, "Convergence properties of a self-adaptive levenberg-marquardt algorithm under local error bound condition," Computational Optimization and Applications., vol. 34, pp. 47-62, 1997.

[26] M. Hagan and M. Menhaj, "Training feedforward networks with the Marquardt algorithm," IEEE Transactions on Neural Networks, vol. 5, no. 6, pp. 989 -993, Nov. 1994.

[27] M. T. Hagan, H. B. Demuth, and M. H. Beale, Neural network design. Boston, London, UK: PWS Pub, 1996.

[28] J. Arif, N. Ray Chaudhuri, S. Ray, and B. Chaudhuri, "Self-tuning feedback linearization controller for power oscillation damping," IEEE PES Transmission and Distribution Conference and Exposition, New Orleans, LA, USA, pp. 1-8, 2010.

[29] O. Malik, G. Chen, G. Hope, Y. Qin, and G. Xu, "Adaptive selfoptimising pole shifting control algorithm," EE Proceedings - Control Theory and Applications, vol. 139, no. 5, pp. 429-438, 1992.

[30] J. W. Chapman, M. D. Ilic, C. A. King, L. Eng, and H. Kaufman, "Stabilizing a multimachine power system via decentralized feedback linearizing excitation control," IEEE Transactions on Power Systems, vol. 8, no. 3, pp. 830-839, 1993.

[31] Y. H. Kim and F. L. Lewis, High-level feedback control with neural networks: World scientific series in robotics and intelligent systems. Singapore, London: World Scientific, 1998.

[32] A. Yesildirek and F. L. Lewis, "Feedback linearization using neural networks," Automatica, vol. 31, no. 11, pp. 1659-1664, 1995.

[33] S. Jagannathan, S. Commuri, and F. L. Lewis, "Feedback linearization using CMAC neural networks," Automatica, vol. 34, pp. 547-557, 1998.

[34] G. Palli, C. Melchiorri, T. Wimbock, M. Grebenstein, and G. Hirzinger, "Feedback linearization and simultaneous stiffness-position control of robots with antagonistic actuated joints," IEEE International Conference on Robotics and Automation, Roma, pp. 4367 - 4372, 2007.

[35] B. Pal and B. Chaudhuri, Robust control in power systems, ser. Power electronics and power systems. New York: Springer, 2005.

[36] N. Martins and L. T. G. Lima, "Determination of suitable locations for power system stabilizers and static VAR compensators for damping electromechanical oscillations in large scale power systems," IEEE Transactions on Power Systems, vol. 5, no. 4, pp. 1455-1469, 1990.

[37] B. Chaudhuri, S. Ray, and R. Majumder, "Robust low-order controller design for multi-modal power oscillation damping using flexible AC transmission systems devices," IET, Generation, Transmission \& Distribution, vol. 3, no. 5, pp. 448-459, 2009.

[38] J. Kennedy and R. Eberhart, "Particle swarm optimization," Proceedings IEEE International Conference on Neural Networks, vol. 4, 1995.

[39] Y. Zhang and S. Ge, "A general recurrent neural network model for time-varying matrix inversion," Proceedings of 42nd IEEE Conference on Decision and Control, vol. 6, pp. 6169 - 6174, Dec. 2003.

[40] V. Kurkov, "Kolmogorov's theorem and multilayer neural networks," Neural Networks, vol. 5, no. 3, pp. 501 - 506, 1992.

[41] A. Albert, Regression and the Moore-Penrose pseudoinverse, ser. Mathematics in science and engineering. New York: Academic Press, 1972.

[42] R. M. Brooks and K. Schmitt, The contraction mapping principle and some applications. Electronic Journal of Differential Equations, Monograph 09, 2009.

[43] K. J. Astrom and B. Wittenmark, Adaptive control, 2nd ed. New York: Addison-Wesley, 1995.

[44] G. C. Goodwin and K. S. Sin, Adaptive filtering prediction and control. Englewood Cliffs, New Jersey: Prentice Hall, 1984. 